\title{
1 WhY DO THEY TRUST IT? DETERMINANTS OF THE INITIAL TRUST IN 2 Automated Driving
}

3

4 J. B. Manchon ${ }^{1,2}$, Romane Beaufort ${ }^{3}$, Mercedes Bueno ${ }^{1}$, and Jordan Navarro 2,4

$5 \quad{ }^{1}$ VEDECOM Institute, Versailles, France

$6 \quad{ }^{2}$ Laboratoire d'Etude des Mécanismes Cognitifs (EA 3082), University Lyon 2, Bron, France

$7 \quad{ }^{3}$ IDUP, Paris, France

$8 \quad{ }^{4}$ Institut Universitaire de France, Paris, France

9

10 Correspondence address: J. B. Manchon, Institut VEDECOM, 23 bis Allée des

11 Marronniers, 78000 Versailles, France; e-mail: jb.manchon@vedecom.fr.

13 Word count: 4102 


\section{HighLIGHTS}

15 - Trust in automated driving should be assessed with specific questionnaires, rather 16 than general ones.

17 - Initial trust in automated driving can be modelled using socio-demographics variables

18 alongside several constructs extracted from trust theoretical models.

19 - Younger people and women seem to trust less automated driving compared to other 20 drivers.

21 - Perceived reputation of automation, proneness to delegate tasks, attitude and 22 expectation of performance are linked with a higher level of trust in automated driving.

23 - Initial trust in automated driving scores can be predicted from the aforementioned 24 constructs. 


\section{ABSTRACT}

26 Because driving automation is quickly increasing, humans' relation to automated driving

27 evolves accordingly. Trust in automated driving has been shown to strongly correlate with

28 drivers' acceptance of such systems. Nonetheless, there is currently a lack of specific

29 questionnaires to assess drivers' level of trust in Highly Automated Driving (HAD).

30 Moreover, little is known about which factors determine trust before any interaction with

31 HAD systems. Theoretical trust in automation models propose several factors to explain

32 how trust builds before and during interactions with automation. Using 844 answers

33 collected through an online survey, this paper aims to propose a new scale designed to

34 evaluate initial trust in automated driving. Moreover, we also measure other factors linked

35 to drivers' initial level of trust in HAD by operationalizing the trust components proposed

36 by Hoff and Bashir (2015)'s theoretical trust model. In order to better describe what

37 determines trust in HAD, a linear model based on the collected data is proposed. The

38 model not only weights the factors determining trust but is also able to predict the level of

39 trust considering these factors.

41 Keywords: trust in automation, trust building, automated driving, prediction, linear model 


\section{INTRODUCTION}

43 The increasing number of low-level automated vehicles on road are changing the way we

44 drive. Tomorrow, Highly Automated Driving (HAD) will deeply modify the relationships

45 between drivers and their automated vehicles (Navarro, 2019).

HAD is defined here as a technology that allows the driver to entirely disengage from the driving task when the HAD system is situated within its operational design domain (level 4, SAE, 2016) or when it does not have an operational design domain anymore (level 5 , SAE, 2016). Nevertheless, for the HAD massive adoption to happen, drivers shall accept to engage in cooperation with such automations. Trust in Automation (TiA) has proved

51 itself to be one of the most predictive factors influencing technology adoption (Kaur \& 52 Rampersad, 2018; Zhang et al., 2019) and use (Dzindolet et al., 2003; Lee \& See, 2004; 53 Rice, 2009).

54 Built on previous research and an extensive literature review, Hoff and Bashir (2015) 55 proposed a general model of factors underlying and empowering TiA. This model 56 proposes a three-layer structure (based on Marsh \& Dibben, 2003) to describe TiA. The 57 first layer, dispositional trust, focuses on individual factors such as age, gender, culture, 58 and personality traits. It is described as a static layer which evolve slowly during an 59 individual's life and has a relatively weak influence on TiA. The second layer, situational 60 trust, designates environmental factors influencing the relation between an operator and 61 the automation. It is composed of two parts corresponding to external and internal 62 variabilities. External factors are related to, e.g., the type of automation, the complexity of 63 the task, the workload, while internal factors refer to the operator's expertise, attentional 64 capacities, mood, and self-confidence. This layer is supposed to evolve faster depending 
65 on the aforementioned factors and have a stronger impact on TiA before the interaction

66 with a particular automation. The third layer, learned trust, is divided in two sublayers: the

67 initial learned trust and the dynamic learned trust. The initial learned trust leans on the

68 operator's pre-existing knowledge about the automation, e.g., the attitudes and

69 expectations, or the past experiences with similar automations. On the other hand, the

70 dynamic learned trust focuses on factors related to the current operator's experience with

71 a particular automation, during use. It includes several factors related to the automation

72 performance and its design.

73 Because it brings a valuable synthesis of its topic, this model has been widely used during

74 the past years and has proved itself to be a strong theoretical frame in the automated

75 driving context (e.g., Ekman et al., 2018; Hergeth et al., 2016; Kraus, Scholz, Messner,

76 et al., 2020; Manchon et al., 2022; Molnar et al., 2018; Walker et al., 2019). Hoff and

77 Bashir's model describes and organizes the factors linked with trust and has been shown

78 to fit for studying TiAD at several steps of the interaction between the driver and the HAD

79 system (see Manchon et al., 2020 for a review). Nevertheless, this relation of trust has

80 common points with the general TiA theory, but also some major particularities that justify

81 the development of another, more specific concept: Trust in Automated Driving (TiAD).

82 The safety critical nature of the driving task is especially consequential because it involves

83 directly the driver and passengers' lives, as well as other people's lives on road. For

84 example, if one uses a HAD system for two years without any incident, a dramatic drop

85 of TiAD can be expected if the automation suddenly fails to prevent a collision with a

86 cyclist. Contrarily, if one is uncomfortable with a HAD system's way to manage speed and

87 steering control, a strong increase in TiAD may be expected if the automation achieves 
88 to prevent a collision with the cyclist. The danger is therefore even more perceptible than

89 it is for other type of dangerous interactions with automation (e.g., powerplant

90 supervision). Because the driving environment is highly variable and likely to evolve

91 drastically in a few seconds, TiAD is prone to a dynamic evolution, including periodical

92 recalibrations within short periods of time. Moreover, the wide range of drivers' individual

93 differences (such as age, abilities, driving experience and driving style, expectations, level

94 of knowledge and understanding) lead to a heterogenous panel of automated driving

95 systems' potential users. This diversity of profiles must therefore be assessed in order to

96 better understand how TiAD builds. Furthermore, no current vehicle is equipped with HAD

97 systems (level 4 or 5, SAE, 2016). The factors related to previous experience and current

98 automation's performance therefore appear irrelevant. On the contrary, factors related to

99 drivers' mental representation of HAD (such as reputation, beliefs, knowledge) seems to

100 be determinant in this context. In the same manner Trust in Robots needed a specific

101 scale to assess more precisely its related factors (Schaefer, 2013), a scale integrating

102 specific factors related to Trust in Automated Driving seemed necessary to investigate

103 this construct. The combination of the aforementioned particularities makes TiAD a

104 specific construct to be assessed with a specific scale.

105 Nonetheless, few studies focused on the determinants underlying drivers' TiAD before 106 interaction with HAD. In an online survey, Kraus et al. (2019) showed that some 107 personality traits had a strong influence on participants' a priori information processing 108 regarding a level 3 (SAE, 2016) automated vehicle. They were able to show respondents' 109 initial TiAD was strongly influenced by the brand reputation and the level of expected 110 reliability of the level 3 driving automation. Ayoub et al. (2021) developed a predictive 
111 model to understand what determines dispositional and initial learned trust. Their online

112 survey included factors such as knowledge, experience, risk and benefit perceptions, and

113 feelings about HAD systems. This model showed a strong effect of the expected risks

114 and benefits, alongside with excitement, knowledge about HAD and eagerness to adopt

115 new technologies. These two models bring valuable information about what determines

116 drivers' initial level of TiAD, but many potential factors related to individuals and

117 automations' characteristics have not been explored yet. To fill this gap, the present

118 contribution proposes to investigate Hoff and Bashir (2015)'s factors linked with

119 dispositional and initial learned trust to better understand their influence on the initial level

120 of TiAD in the population.

121 The goals of this paper are therefore (a) to propose a new scale designed to assess trust

122 in the specific context of driving automation and (b) to participate to fill the gap in the

123 understanding of the initial level of TiAD, by assessing the numerous factors related to

124 individuals (i.e., dispositional trust and initial learned trust, Hoff \& Bashir, 2015), and

125 proposing a model integrating the respective weights of these factors. 


\section{METHOD}

\section{Participants and procedure}

128 The questionnaire was sent by email and several social media during summer 2020 to a

129 large panel of people. The only criterion was to speak French, for participants to be able

130 to understand the questions. Participants' socio-demographics data are reported in Table

$131 \quad 2$ and the procedure is displayed in Figure 1.

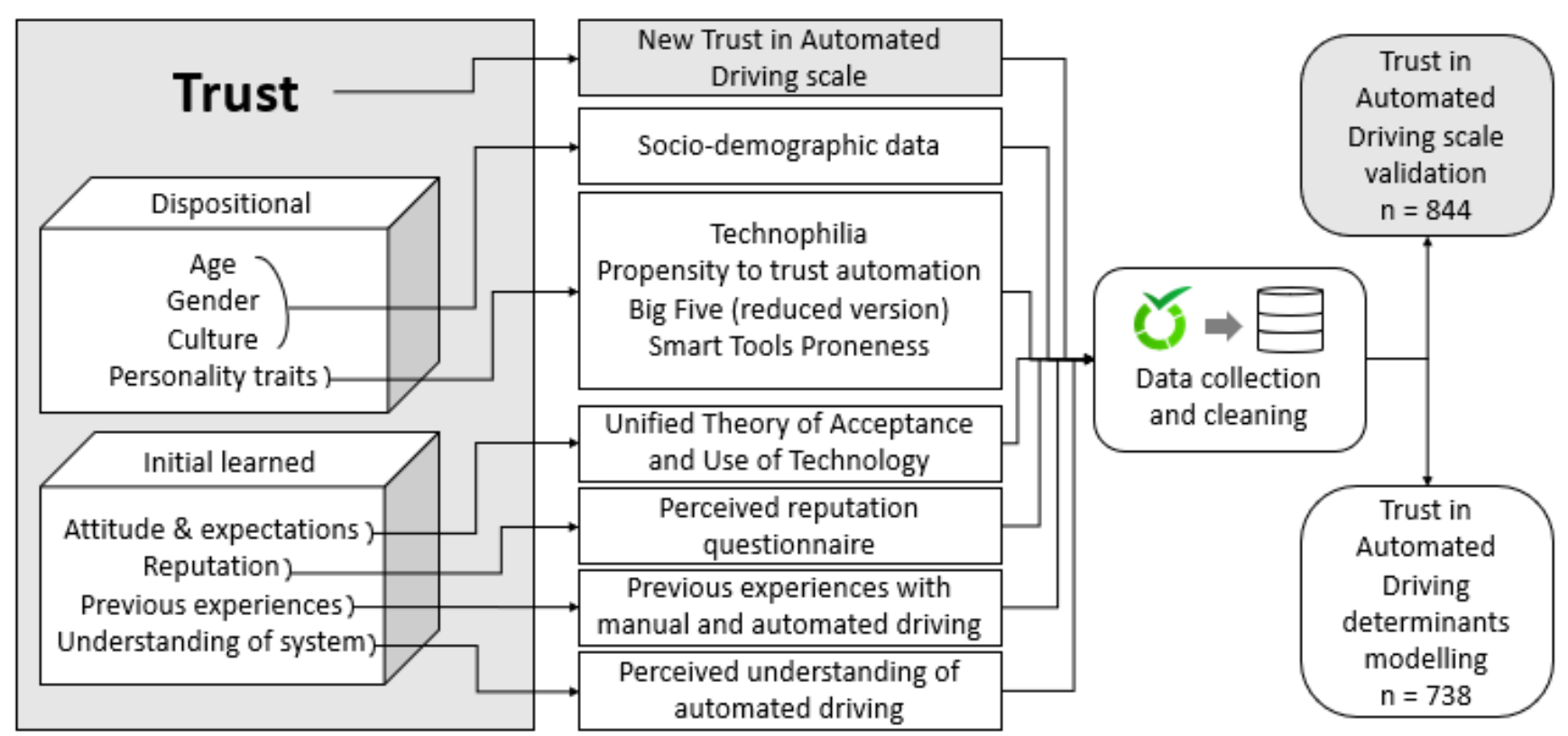

Figure 1. Data collection procedure. The left part represents the trust factors from Hoff and Bashir (2015).

The center part presents the questionnaires that were used for the data collection. The right part displays the targeted outputs.

132 In the first part of the present contribution, a new 10-item TiAD scale was developed and

133 assessed to inquire its internal validity and reliability. A total of 844 participants answered

134 this 10-item TiAD scale, which was the first step of the overall questionnaire (Figure 1).

135 In the second part of the contribution, the answers of 738 participants who had completed

136 the full questionnaire were kept modelling the determinants of TiAD using the 10-item

137 scale and the several other scales (Figure 1). 


\section{Trust scale}

139 The present trust scale was created to measure the initial level of TiAD, in contrast with

140 the dynamic level of TiAD that appears during the interactions between the driver and the

141 HAD system. A careful literature review (Manchon et al., 2020) led to selecting 10 items

142 inquiring trust and adapting them to the HAD context. Items were selected to minimize

143 redundancy across dimensions. Items one, four and ten question general feelings about

144 perceived safety and trust, that are central in the driving context and create the need for

145 a dedicated scale. The seven other items are indirect measures of trust (i.e., comparison

146 with manual driving, complex situation management, weather-related attitude,

147 engagement in non-driving-related activities, boredom-related attitude, and tiredness-

148 related attitude). These items explore participant's expectations and mental models about

149 HAD, which lean on participants beliefs and knowledge about HAD, but also past

150 experiences (with, e.g., advanced driving assistance systems if they have ever used one)

151 and on overall HAD systems' reputation. The investigated questionnaire has been used

152 in past experiments (Manchon et al., 2021, 2022) and has shown a strong correlation with

153 objective trust measure (e.g., gaze behaviour, engagement in non-driving-related

154 activities, Manchon et al., 2020). Items are shown in Table 1 and participants'

155 demographic data are displayed in Table 2.

156 Each item response ranged from 1 to 6 , and the total trust score was obtained by adding

157 each item's value, reversing item 3 (Table 1). Trust scores were then converted in

158 percentage by subtracting 10 from this total sum to make it range from 0 to 50 . They were

159 then transformed using the rule of three (i.e., dividing them by their theoretical maximum,

16050 , and multiplying them by their targeted maximum, 100). The formula, using $s_{t}$ for the 
161 total trust score and $s_{n}$ for each item's value was $s_{t}=\left(s_{1}+s_{2}+\left(7-s_{3}\right)+s_{4}+s_{5}+s_{6}+\right.$ $\left.162 s_{7}+s_{8}+s_{9}+s_{10}-10\right) \div 50 \times 100$. The scores used in further analysis were therefore

163 linear conversions of the simple total sums, to ease readers' assessments of values by

164 providing percentages.

Table 1. Trust in Automated Driving measurement scale

\begin{tabular}{|c|c|c|}
\hline & Questions & Sources \\
\hline 1 & I would feel safe in an automated vehicle. & $\begin{array}{l}\text { O'Cass and } \\
\text { Carlson (2012) }\end{array}$ \\
\hline 2 & $\begin{array}{l}\text { The automated driving system provides me with more safety compared } \\
\text { to manual driving. }\end{array}$ & Payre et al. (2016) \\
\hline $3^{*}$ & $\begin{array}{l}\text { I would rather keep manual control of my vehicle than delegate it to the } \\
\text { automated driving system on every occasion. }\end{array}$ & Payre et al. (2016) \\
\hline 4 & I would trust automated driving system decisions. & $\begin{array}{l}\text { O'Cass and } \\
\text { Carlson (2012) }\end{array}$ \\
\hline 5 & $\begin{array}{l}\text { I would trust automated driving system capacities to manage complex } \\
\text { driving situations. }\end{array}$ & $\begin{array}{c}\text { Egea and } \\
\text { González (2011) }\end{array}$ \\
\hline 6 & $\begin{array}{l}\text { If the weather conditions were bad (e.g., fog, glare, rain), I would } \\
\text { delegate the driving task to the automated driving system. }\end{array}$ & Payre (2015) \\
\hline 7 & $\begin{array}{l}\text { Rather than monitoring the driving environment, I could focus on other } \\
\text { activities confidently. }\end{array}$ & $\begin{array}{l}\text { Egea and } \\
\text { González (2011) }\end{array}$ \\
\hline 8 & $\begin{array}{l}\text { If driving was boring for me, I would rather delegate it to the automated } \\
\text { driving system than do it myself. }\end{array}$ & Payre et al. (2016) \\
\hline 9 & $\begin{array}{l}\text { I would delegate the driving to the automated driving system if I were } \\
\text { tired. }\end{array}$ & Payre et al. (2016) \\
\hline 10 & How much do you trust automated driving systems? & $\begin{array}{l}\text { Lee and Moray } \\
\text { (1994) }\end{array}$ \\
\hline
\end{tabular}


Table 2. Participant's socio-demographics data for the trust scale validation.

\begin{tabular}{|c|c|c|c|c|c|c|}
\hline & \multicolumn{3}{|l|}{$\mathrm{n}$} & \multicolumn{3}{|c|}{ Frequency (\%) } \\
\hline Total & \multicolumn{3}{|l|}{844} & \multicolumn{3}{|l|}{$100 \%$} \\
\hline \multirow[t]{2}{*}{ Female } & \multicolumn{3}{|l|}{448} & \multicolumn{3}{|l|}{$53.10 \%$} \\
\hline & Min & 1st Q & Med & Mean & 3rd Q & $\operatorname{Max}$ \\
\hline Age (years) & 16 & 29 & 40 & 40.47 & 51 & 93 \\
\hline Driver's licence since (years) & 0 & 10 & 21 & 21.89 & 32 & 73 \\
\hline \multicolumn{7}{|l|}{ Driving frequency } \\
\hline Every day or almost & \multicolumn{3}{|l|}{412} & \multicolumn{3}{|l|}{$48.82 \%$} \\
\hline Several times a week & \multicolumn{3}{|l|}{170} & \multicolumn{3}{|c|}{$20.14 \%$} \\
\hline Several times a month & \multicolumn{3}{|l|}{92} & \multicolumn{3}{|l|}{$10.90 \%$} \\
\hline Less often & \multicolumn{3}{|l|}{93} & \multicolumn{3}{|l|}{$11.02 \%$} \\
\hline No driving licence & \multicolumn{3}{|l|}{77} & \multicolumn{3}{|l|}{$9.12 \%$} \\
\hline \multicolumn{7}{|l|}{ Kilometres driven by year } \\
\hline Less than $5,000 \mathrm{~km}$ & \multicolumn{3}{|l|}{208} & \multicolumn{3}{|l|}{$24.64 \%$} \\
\hline Between 5,000 and $10,000 \mathrm{~km}$ & \multicolumn{3}{|l|}{214} & \multicolumn{3}{|c|}{$25.36 \%$} \\
\hline Between 10,000 and $20,000 \mathrm{~km}$ & \multicolumn{3}{|l|}{214} & \multicolumn{3}{|c|}{$25.36 \%$} \\
\hline Between 20,000 and $50,000 \mathrm{~km}$ & \multicolumn{3}{|l|}{131} & \multicolumn{3}{|c|}{$15.52 \%$} \\
\hline \multicolumn{7}{|l|}{ Education } \\
\hline None & \multicolumn{3}{|l|}{4} & $0.47 \%$ & & \\
\hline GCSE & 6 & & & $0.71 \%$ & & \\
\hline BTEC National Diploma & 30 & & & $3.55 \%$ & & \\
\hline A-levels & 138 & & & $16.35 \%$ & & \\
\hline HND or Two years degree & 202 & & & $23.93 \%$ & & \\
\hline Bachelor's degree or Three years degree & 96 & & & $11.37 \%$ & & \\
\hline $\begin{array}{l}\text { Bachelor's with Honours degree or Four } \\
\text { years degree }\end{array}$ & 70 & & & $8.29 \%$ & & \\
\hline Master's degree or Five years degree & 244 & & & $28.91 \%$ & & \\
\hline PhD or Eight years degree & 54 & & & $6.40 \%$ & & \\
\hline Socio-economic Classification & & & & & & \\
\hline Higher grade occupations & 360 & & & $42.65 \%$ & & \\
\hline Intermediate occupations & 53 & & & $6.28 \%$ & & \\
\hline Employees & 285 & & & $33.77 \%$ & & \\
\hline Students & 109 & & & $12.91 \%$ & & \\
\hline Retired people & 37 & & & $4.38 \%$ & & \\
\hline
\end{tabular}




\section{Other constructs and other scales}

168 In order to investigate the determinants of TiAD, other scales were used to measure 169 related constructs, following the Hoff and Bashir (2015)'s model. Whenever it was 170 possible, well-accepted and well-standardized scales were used. In several cases, proxy

171 variables were used to approach some Hoff and Bashir (2015)'s factors. For example, 172 because of a lack of funding, culture could not be assessed with multi-language

173 questionnaire broadcasted in several countries. Culture was therefore inquired through 174 socio-professional category, level of diploma, marital status, and parenthood. The 175 complete inventory of constructs is listed in Table 3.

Table 3. Measured constructs and related scales.

\begin{tabular}{|c|c|c|c|}
\hline Measured constructs & $\begin{array}{l}\text { Number } \\
\text { of items }\end{array}$ & $\begin{array}{l}\text { Scale } \\
\text { type }\end{array}$ & Reference \\
\hline \multicolumn{4}{|c|}{ Hoff and Bashir (2015)'s dispositional trust } \\
\hline Age & 1 & & \\
\hline Gender & 1 & & \\
\hline \multicolumn{4}{|l|}{ Culture } \\
\hline Socio-Professional Category & 1 & Custom & $\begin{array}{l}\text { Desrosières and } \\
\text { Thévenot (2002) }\end{array}$ \\
\hline Diploma & 1 & Custom & New item \\
\hline Marital status & 1 & Custom & New item \\
\hline Parenthood & 1 & Custom & New item \\
\hline \multicolumn{4}{|l|}{ Personality Traits } \\
\hline Technophilia questionnaire & 4 & $\begin{array}{c}\text { 7-point } \\
\text { Likert } \\
\text { type }\end{array}$ & $\begin{array}{l}\text { Agarwal and } \\
\text { Prasad (1998) }\end{array}$ \\
\hline Propensity to trust technology questionnaire & 6 & $\begin{array}{c}\text { 7-point } \\
\text { Likert } \\
\text { type }\end{array}$ & $\begin{array}{l}\text { Schneider et al. } \\
\text { (2017) }\end{array}$ \\
\hline Big Five Inventory (French reduced adaptation) & 45 & \multirow{6}{*}{$\begin{array}{c}\text { 5-point } \\
\text { Likert } \\
\text { type }\end{array}$} & \\
\hline Extraversion & 8 & & \multirow{5}{*}{$\begin{array}{l}\text { Plaisant et al. } \\
\text { (2010) }\end{array}$} \\
\hline Agreeableness & 10 & & \\
\hline Conscientiousness & 9 & & \\
\hline Neuroticism & 8 & & \\
\hline Openness & 10 & & \\
\hline Smart Tools Proneness Questionnaire (STP-Q) & 27 & \multirow{3}{*}{$\begin{array}{l}\text { 7-point } \\
\text { Likert }\end{array}$} & \\
\hline Smart tools performances and efficiency & 12 & & \multirow{2}{*}{$\begin{array}{l}\text { Navarro et al. } \\
\text { (submitted) }\end{array}$} \\
\hline Social acceptance and smart tools personal & 8 & & \\
\hline
\end{tabular}




\begin{tabular}{|c|c|c|c|}
\hline suitability & & \multirow[t]{2}{*}{ type } & \\
\hline Proneness to delegate tasks & 7 & & \\
\hline \multicolumn{4}{|c|}{ Hoff and Bashir (2015)'s initial learned trust } \\
\hline $\begin{array}{l}\text { Unified Theory of Acceptance and Use of Technol- } \\
\text { ogy (UTAUT) }\end{array}$ & 12 & & \\
\hline Attitude & 4 & \multirow{3}{*}{$\begin{array}{l}\text { 6-point } \\
\text { Likert } \\
\text { type }\end{array}$} & \multirow{3}{*}{$\begin{array}{l}\text { Adapted from } \\
\text { Venkatesh et al } \\
(2003)\end{array}$} \\
\hline Expectation of performance & 4 & & \\
\hline Expectation of utility & 4 & & \\
\hline Perceived reputation of automated driving systems & 4 & & \\
\hline General reputation of HAD systems & 1 & \multirow{4}{*}{$\begin{array}{l}\text { 6-point } \\
\text { Likert } \\
\text { type }\end{array}$} & \multirow{4}{*}{ New scale } \\
\hline $\begin{array}{l}\text { Reputation of HAD systems designers } \\
\text { (capabilities) }\end{array}$ & 1 & & \\
\hline $\begin{array}{l}\text { Reputation of cars manufacturers selling HAD } \\
\text { systems (reliability) }\end{array}$ & 1 & & \\
\hline $\begin{array}{l}\text { Reputation of institutional actors promoting } \\
\text { HAD system (general interest of public policies) }\end{array}$ & 1 & & \\
\hline $\begin{array}{l}\text { Previous experiences with automated driving } \\
\text { systems }\end{array}$ & 3 & & \\
\hline As a driver in a personal car & 1 & Custom & New scale \\
\hline As a passenger in a personal car & 1 & Custom & New scale \\
\hline $\begin{array}{l}\text { As a passenger in an automated shuffle or } \\
\text { another public transport automated vehicle }\end{array}$ & 1 & Custom & New scale \\
\hline $\begin{array}{l}\text { Self-evaluated understanding and knowledges } \\
\text { about automated driving systems }\end{array}$ & 1 & Custom & New scale \\
\hline \multicolumn{2}{|l|}{ Driving experience } & & \\
\hline $\begin{array}{l}\text { Number of years since the obtention of a driving } \\
\text { licence }\end{array}$ & 1 & & \\
\hline Driving frequency & 1 & Custom & \\
\hline
\end{tabular}

Data analysis

177 In the next section, the 844 scores from the trust scale have been examined through

178 exploratory factor analysis (EFA) and confirmatory factor analysis (CFA) in order to

179 investigate the structure and properties of the proposed trust scale. Then, 738 complete

180 answers have been used to yield a linear model integrating the principal determinants of

181 TiAD, considering the other constructs linked to trust by Hoff and Bashir (2015). This linear

182 model has then been analysed to weight the relative influence of each construct on the

183 participants' level of TiAD. To do so, each construct have been scored in accordance with 
184 authors recommendations, then rescaled to range from 0 to 100 . This conversion was

185 done to ease interpretations. To construct the linear model, data were standardized.

\section{3. RESULTS}

187 Trust scale validation

188 The properties of the trust in automated driving scale were analysed using $R$ 3.6.1 ( $R$

189 Core Team, 2017), the psych (Revelle, 2020) and the lavaan packages (Rosseel, 2012).

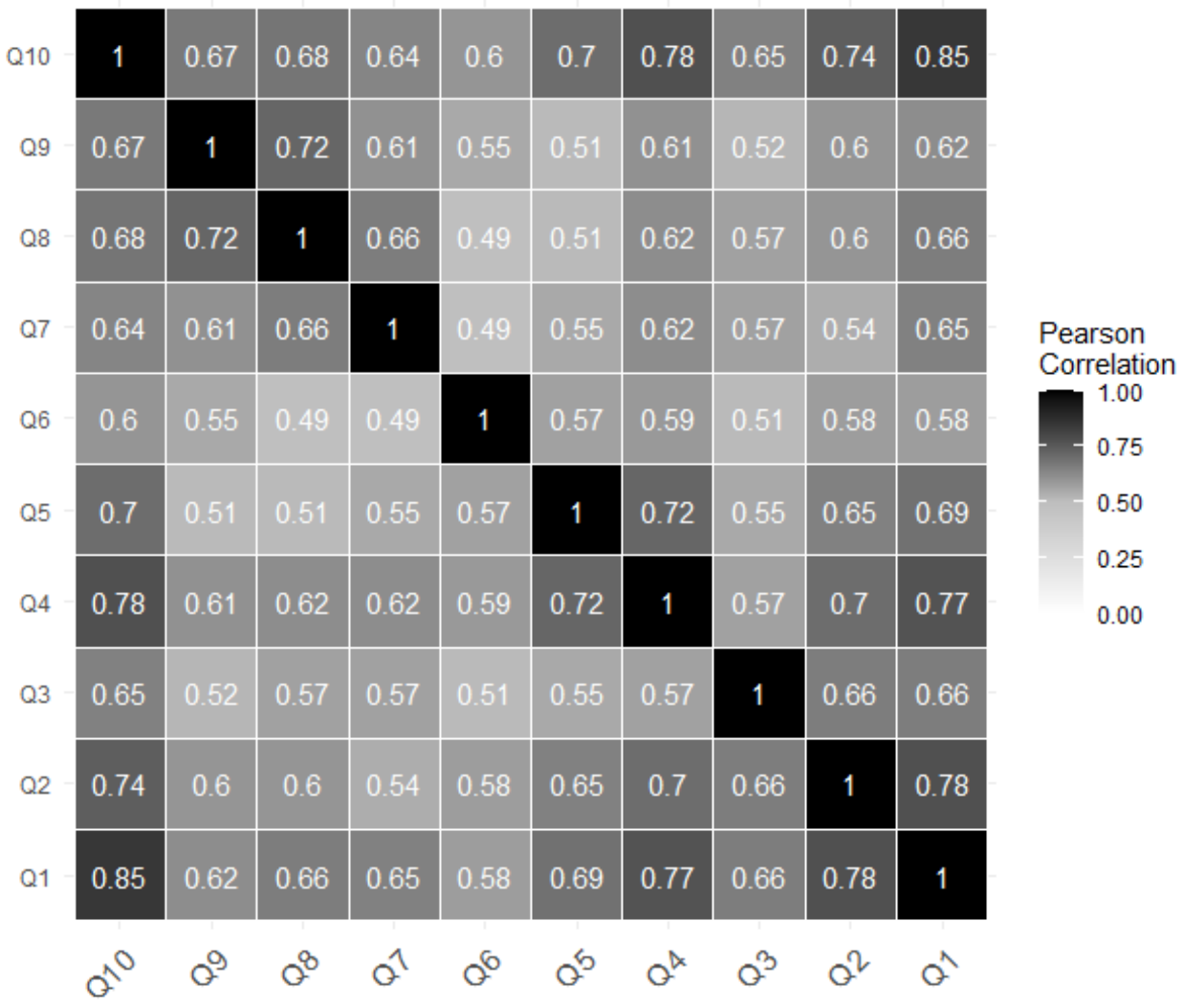

Figure 2. Correlation matrix of the scales' items. All correlations are significant at $p<$ 0.001 using Benjamini \& Hochberg (1995)'s correction for multiple comparisons.

190 Homogeneity and internal consistency were good (Cronbach's $\alpha=.94$, McDonald's $\omega_{\mathrm{h}}$ $191=.78$, and $\left.\omega_{\mathrm{t}}=.96\right)$. The correlation matrix of each item's score is displayed in Figure 2. 192 It shows all items are strongly related. 
193 Data were appropriate for a factor analysis, according to the measure of sampling 194 adequacy $(\mathrm{KMO}=0.95)$. Furthermore, the Bartlett's test of sphericity confirmed the 195 number of significant correlations was sufficient for a factor analysis $\left(X^{2}(45)=6725.04\right.$, $196 p<.001)$. The use of a single factor structure for the 10-item scale was supported by 11 197 out of the $23(47.83 \%)$ methods used to determine the optimal number of factors (i.e., $t$, 198 , Optimal coordinates, Acceleration factor, Parallel analysis, Kaiser criterion, SE Scree, 199 R2, VSS complexity 1, Velicer's MAP, and RMSEA). Following Revelle (2020)'s proposed 200 procedure, exploratory factor analysis (EFA) was then applied to investigate the scale's 201 factor structure. A maximum likelihood factor analysis using varimax rotation was used to 202 study this single factor (i.e., trust), following Costello and Osborne (2005)'s 203 recommendations. The EFA showed that a one-dimensional factor accounted for $62.92 \%$ 204 of the total variance of the data. EFA loadings are displayed in Table 4. A confirmatory 205 factor analysis (CFA) was then produced to confirm the EFA results. Seventy percent of 206 the data were used for the CFA training and the remaining thirty percent for testing. The 207 following model was yielded: $X^{2}=125.1, d f=35, p<.001$, AGFI $=.86$ (good: $\geq .85$ ), 208 CFI $=.95$ (good: $\geq .85$ ), TLI $=.92$ (good: $\geq .80$ ), RMSEA $=.10$ (acceptable: $\leq .10$, $209 \mathrm{Cl}$ low $=.08 ; \mathrm{Cl}$ high $=0.12)$ and $\mathrm{SRMR}=.04$ (good: $\leq .05)$. Details for each item are 210 given in Table 4. The path weight corresponding to this CFA are displayed in Figure 3. 211 Considering the aforementioned indicators, the model seemed good according to Brown 212 (2015)'s recommendations. The scale can therefore be confidently used in further 213 analyses. 
Table 4. Loadings for the EFA and CFA related to the TiAD measurement scale

\begin{tabular}{|c|ccc|cccc|}
\hline \multirow{2}{*}{ Items } & \multicolumn{4}{|c|}{ EFA } & \multicolumn{4}{c|}{ CFA } \\
\cline { 2 - 8 } & Loadings & Complexity & Uniqueness & Estimate & $\begin{array}{c}\text { Standard } \\
\text { Error }\end{array}$ & z-value & $p$ \\
\hline Q1 & 0.91 & 1.00 & 0.18 & 1.000 & & & \\
\hline Q2 & 0.83 & 1.00 & 0.31 & 0.977 & 0.047 & 20.654 & $<0.001$ \\
\hline Q3 & 0.73 & 1.00 & 0.47 & 0.897 & 0.063 & 14.154 & $<0.001$ \\
\hline Q4 & 0.85 & 1.00 & 0.27 & 0.871 & 0.044 & 19.870 & $<0.001$ \\
\hline Q5 & 0.77 & 1.00 & 0.41 & 0.831 & 0.050 & 16.476 & $<0.001$ \\
\hline Q6 & 0.68 & 1.00 & 0.54 & 0.844 & 0.066 & 12.835 & $<0.001$ \\
\hline Q7 & 0.73 & 1.00 & 0.47 & 0.833 & 0.060 & 13.853 & $<0.001$ \\
\hline Q8 & 0.76 & 1.00 & 0.43 & 0.853 & 0.060 & 14.322 & $<0.001$ \\
\hline Q9 & 0.74 & 1.00 & 0.46 & 0.800 & 0.058 & 13.824 & $<0.001$ \\
\hline Q10 & 0.91 & 1.00 & 0.17 & 0.913 & 0.038 & 24.193 & $<0.001$ \\
\hline
\end{tabular}

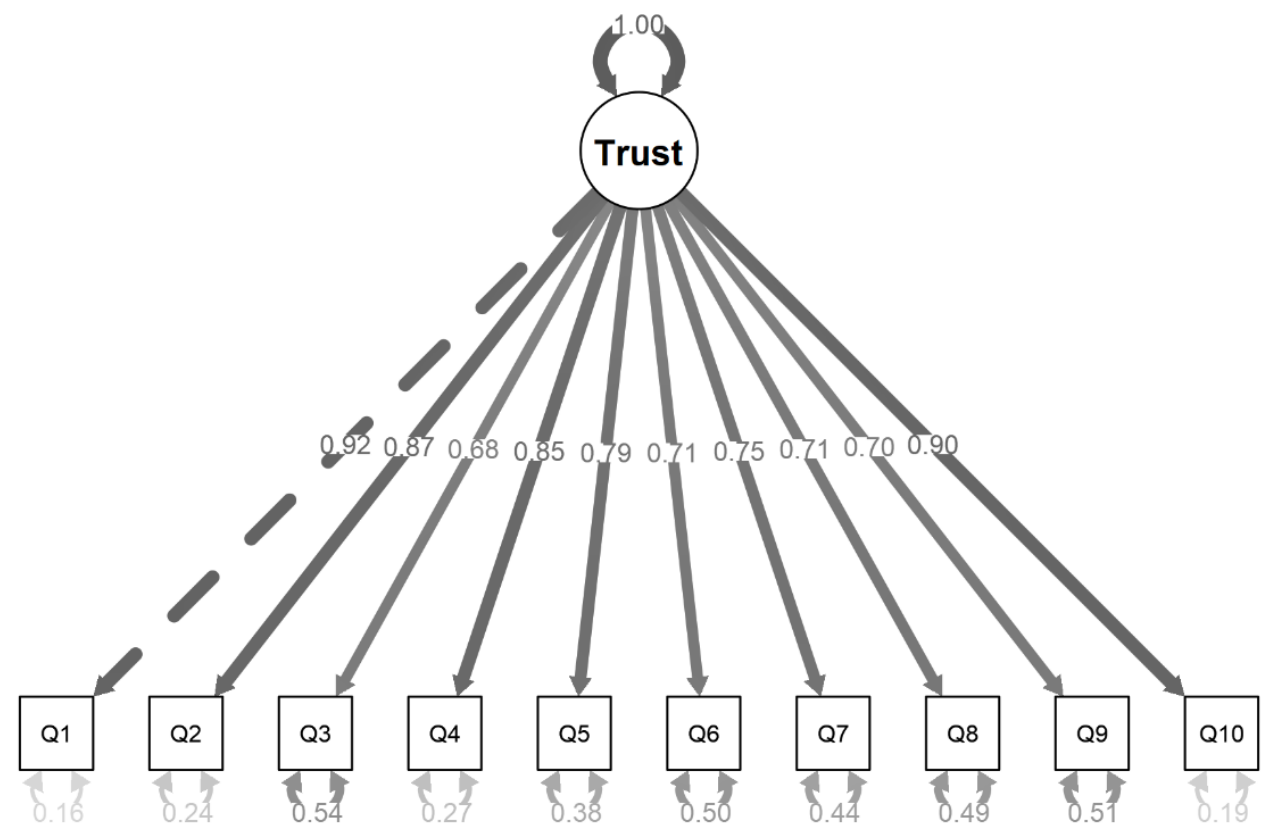

Figure 3. Path weights calculated from the CFA.

\section{Linear model construction}

215 The linear model describing trust through several related constructs was created using $R$

216 3.6.1 (R Core Team, 2017). The correlation matrix of the variables and constructs is 
217 displayed in Figure 4. This matrix shows that numerous variables are related all together, 218 confirming the need for a model that untangle these complex relations.

219 The construction of the models led to drop variables that were showing strong collinearity 220 and were therefore redundant (e.g., age and number of years since the obtention of a 221 driving licence). Several other variables appeared to have no specific effect (i.e., all things 222 being equal, technophilia was mostly explained by attitude and added no more 223 information) and were removed from the model to increase its performance and ease the 224 interpretations. This iterative process was repeated, and temporary models were 225 compared to select the best one. The final model is presented in Table 5. 


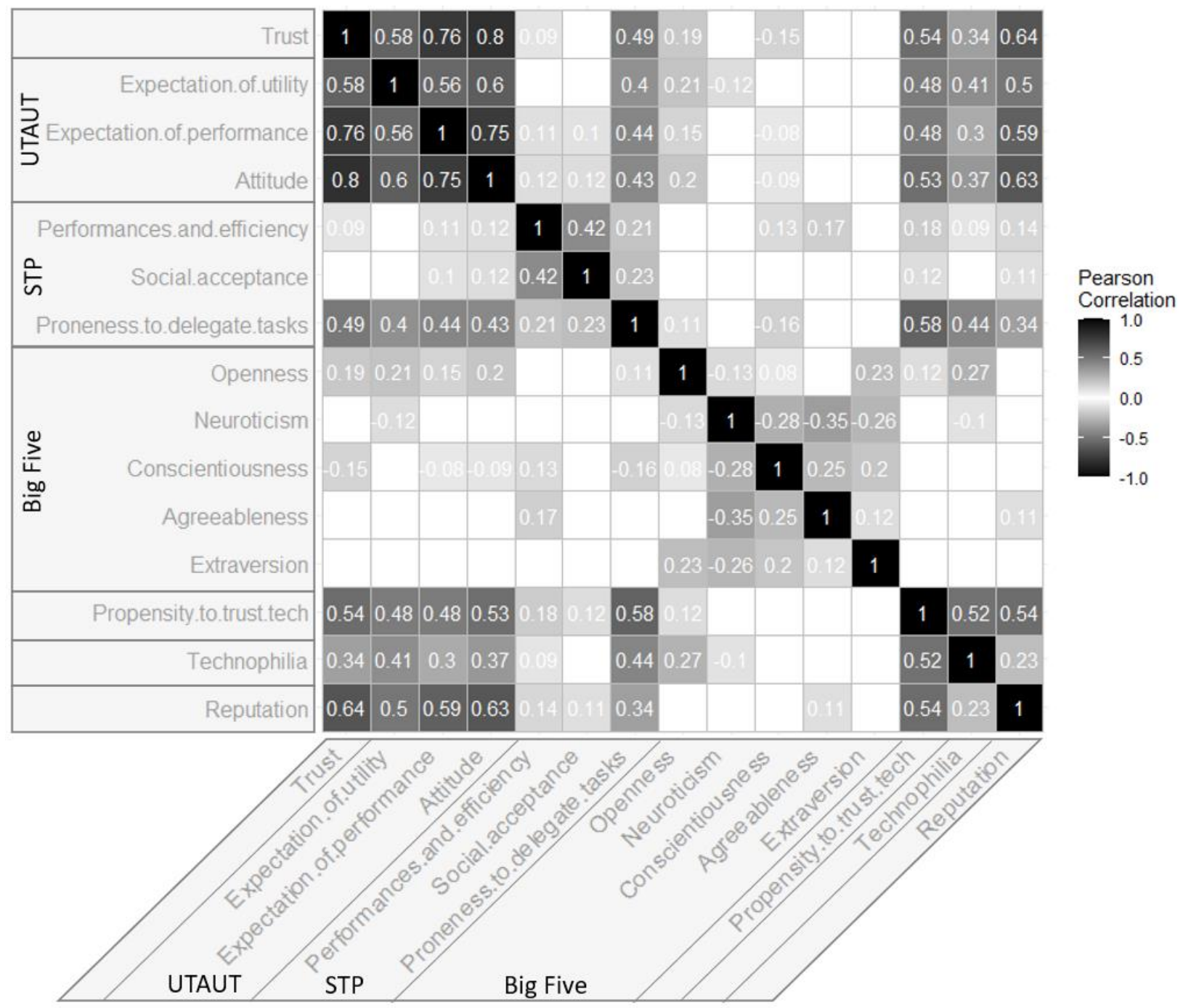

Figure 4. Correlation matrix of the variables and constructs. All displayed correlations are significant at $p<0.05$ using Benjamini \& Hochberg (1995)'s correction for multiple comparisons.

226 Considering factors linked with the dispositional TiAD, the model exhibited an increase in 227 TiAD when age grown (ten years increase TiAD by 1 point, all things equal otherwise), 228 alongside an effect of the gender, where female trusted less HAD systems than male (4.6 229 points less, all things equal otherwise). Higher education also seemed to have a positive 230 effect on TiAD, which was not significant (each supplemental year increase TiAD by 0.5 231 points, all things equal otherwise). Regarding personality traits, the proneness to delegate 232 tasks from the STP-Q showed a positive effect on TiAD, making TiAD increase of 0.16 
233 points for each declared point. One dimension from the Big Five (i.e., conscientiousness)

234 showed a very small negative effect on TiAD.

235 Considering factors linked with initial learned TiAD, the model showed a negative effect 236 of the driving frequency that increased when participants drove more frequently, 237 compared to participants that drove less frequently. Two constructs from the UTAUT 238 (Venkatesh et al., 2003) had a significative influence on TiAD: 1 point of attitude made 239 TiAD increase of 0.4 points, while 1 point of expectation of performances made TiAD 240 increase of 0.3 points. Perceived HAD systems' reputation also had a positive effect, 241 making TiAD increase by 0.2 points for each declared point.

Table 5. Final linear model including variables and constructs linked to TiAD.

Adjusted $R^{2}=.74, F=176.9, D F=12 \& 725$.

\begin{tabular}{|c|c|c|}
\hline Variables and constructs & Estimate $p$ & $p$ \\
\hline (Intercept) & 4.404 & . \\
\hline \multicolumn{3}{|c|}{ Factors linked with dispositional TiAD } \\
\hline Age (in years) & $0.109 *$ & $*$ \\
\hline Female & $-4.588 *$ & $* * *$ \\
\hline Higher education (in years) & 0.503. & . \\
\hline $\begin{array}{l}\text { STP (Proneness to delegate } \\
\text { tasks) }\end{array}$ & $0.099 *$ & $* * *$ \\
\hline \multicolumn{3}{|l|}{ Big Five } \\
\hline Conscientiousness & $-0.049 *$ & $*$ \\
\hline \multicolumn{3}{|c|}{ Factors linked with initial learned TiAD } \\
\hline \multicolumn{3}{|l|}{ Driving frequency } \\
\hline Every day & $-5.230 *$ & $* * *$ \\
\hline Every week & -2.945 & . \\
\hline Less than every week & Reference & \\
\hline \multicolumn{3}{|l|}{ UTAUT } \\
\hline Attitude & $0.441 *$ & $* * *$ \\
\hline Expectation of performance & $0.262 *$ & $* * *$ \\
\hline Perceived reputation & $0.167 *$ & $* * *$ \\
\hline
\end{tabular}




\section{Linear model predictions}

243 The previously presented model can also be used to make predictions, considering all

244 the variables that were included. To test the predictive performances of this model, it was

245 compared to a random TiAD score assignment, and three other models. One integrates

246 only socio-demographic data (i.e., age, gender, diploma, socio-professional category,

247 marital status, parenthood, number of years since the obtention of the driving licence,

248 driving frequency); one integrates all the variables related to dispositional trust; and one

249 integrates all the variables related to initial learned trust. The deviation between observed

250 and predicted TiAD scores from the random assignment and the four models are

251 presented in Figure 5.

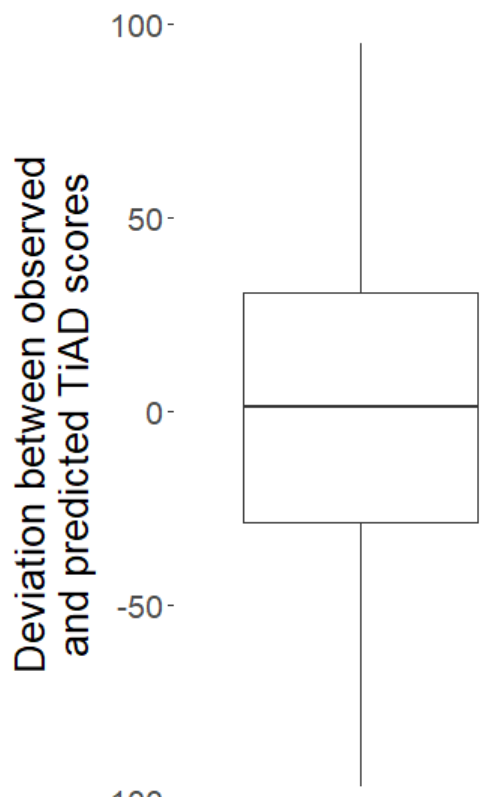

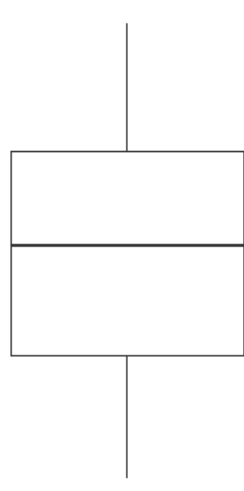

Random

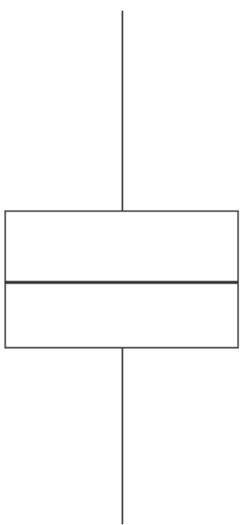

Dispositional trust variables

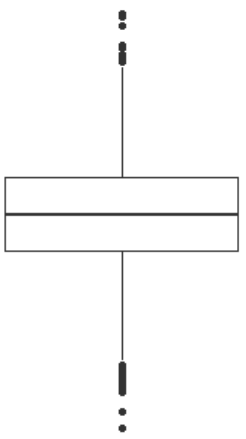

Initial learned trust variables

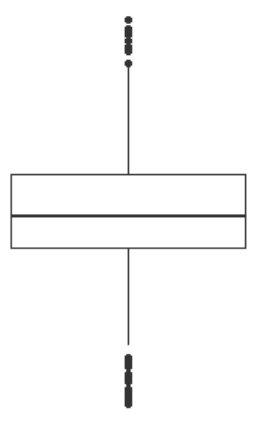

Previousely constructed model

Models

Figure 5. Boxplots of the deviation between observed and predicted TiAD scores from three models.

Random: $\min =-5.10, Q 1=-0.98$, med $=0.01$, mean $=-0.01, Q 3=0.96$, max $=4.96$.

Socio-demographic: $\min =-2.25, Q 1=-0.7$, med $=0.01$, mean $=0.00, Q 3=0.73$, ax $=2.14$.

Final model: $\min =-1.87, Q 1=-0.30$, med $=0.03$, mean $=0.00, Q 3=0.35$, ax $=1.63$. 
252 The results show that using only socio-demographic data to predict TiAD strongly reduces

253 the number of outliers, compared to a random assignment. The random model has an

254 average deviation of 34.3 points of TiAD score, compared to observed data.

255 Comparatively, the socio-demographic model has an average deviation of 25.8 points,

256 which is 8.5 points less. The prediction effectively improves with the dispositional model,

257 which has an average deviation of 20.9 points of TiAD score (4.9 points less), compared

258 to observed data. The initial learned model is better, with an average deviation of 12.3

259 points of TiAD score (8.6 points less) and a lower number of outliers. The previously

260 constructed model is the best one, with an average deviation of 12.2 points of TiAD score.

261 This final model has therefore an average deviation of 22.1 points less than the random

262 model, 13.6 points less than the socio-demographic model, 8.7 points less than the

263 dispositional model, and 0.1 points less than the initial learned model. These results

264 indicate the proposed model brings valuable information, compared with simple socio-

265 demographic data or variables related to the dispositional trust. They also indicate the

266 variables related to the initial learned trust are determinant and may have a good

267 prediction capability even considered alone.

\section{4. Discussion}

269 The present paper had two main goals : (a) to propose a new specific scale to measure

270 the initial level of TiAD; and (b) to use this scale in a large-scale online survey aiming to

271 assess several factors linked with TiAD. The resulting answers were used to construct a

272 linear model indicating what constructs were the most influential on trust. The model was

273 then used to predict trust using different information. 


\section{Initial level of TiAD assessment}

275 A previous literature review (Manchon et al., 2020) initiated a conceptual differentiation 276 regarding the nature of TiAD, compared to the TiA theory that was developed during the 277 last decades. The present contribution aims to extends this theoretical work, proposing a 278 new scale specifically designed to assess TiAD, contrary to the very often used Jian et al. 279 (2000)'s scale that inquire the general TiA. Previous experiments showed this scale was 280 able to measure variation in trust before and after drivers had a simulated experience with 281 a HAD system. Moreover, the results complied with other measures linked with trust, such 282 as visual strategies and engagement in non-driving related activities (Manchon et al., 283 2021, 2022). These results were consistent with the literature (e.g., Hartwich et al., 2018; 284 Hergeth et al., 2017; Körber et al., 2018; Kraus, Scholz, Stiegemeier, et al., 2020), and 285 led to further investigation about this scale's properties. The present results from the EFA 286 and the CFA confirmed this new scale is relevant to assess TiAD. The numerous 287 indicators of the resulting model exhibited the scale's properness to measure the single 288 factor (i.e., trust) it was designed to evaluate. Future studies could compare results from 289 this scale and Jian et al. (2000)'s scale to extend knowledge regarding its ability to assess 290 TiAD.

\section{Determinants of the dispositional TiAD}

292 The construction of a linear model explaining which factors are the most relevant to 293 explain TiAD provided valuable findings.

294 Regarding variables linked with Hoff and Bashir (2015)'s dispositional trust, increasing 295 age showed a very small positive effect on initial TiAD. Previous papers reported elderly 296 people were sometimes interested in HAD because it was a solution to their decreasing 
297 driving skills (Körber et al., 2016; Shin et al., 2019), an explanation consistent with our

298 results. In the same manner, the model indicates females have lower level of initial TiAD,

299 compared to male. This effect of the gender is usually absent in experiments, and this

300 finding raises a few questions. It might exist a gender effect in the overall population, as

301 indicated by Ayoub et al. (2021)'s model, that fades away in experimental sessions

302 because some females that distrust HAD are not interested in participating in this kind of

303 experiments. This finding should be examined thoroughly before even being considered.

304 Personality traits such as the proneness to delegate tasks showed a positive effect on

305 TiAD, which is consistent with the construct (Navarro et al., submitted). Agreeableness,

306 which include a trust subfactor, is usually positively correlated with trust in other human.

307 The negative link between agreeableness and TiAD in the present model is therefore

308 surprising. Additional studies are needed to better understand how general trust differ

309 from TiAD (i.e., how trusting a machine differ from trusting a human), and what other

310 factors are overwhelming the effect of this personality trait. Without surprise,

311 conscientiousness had a negative impact on TiAD. This is consistent with Ayoub et al.

312 (2021)'s model, where participants who feel in control during manual driving show a lower

313 level of TiAD.

\section{Determinants of the initial learned TiAD}

315 Regarding the variables linked with Hoff and Bashir (2015)'s initial learned trust, it seems

316 that experience with manual driving (i.e., driving frequency) had a strong impact on trust.

317 Daily commuters showed a lower level of TiAD, compared to people that drive few times

318 a week, and these weekly drivers also had a lower level of TiAD than casual drivers. As

319 reported by Ayoub et al. (2021), the number of years of driving also seemed to have a 
320 negative impact on TiAD. It is possible that frequent drivers have developed a high level 321 of confidence in their own driving skills and thus tend to trust driving automation less, in 322 line with the seminal findings reported by Lee and Moray (1994).

323 Constructs from the UTAUT (Venkatesh et al., 2003) were highly correlated with TiAD. In 324 particular, attitude was a powerful predictor of TiAD, alongside the expectation of 325 performance. These two constructs were so self-explaining they hided the effects of other 326 constructs that were less correlated, like technophilia or propensity to trust automation. 327 This finding reinforces the idea that these questionnaires are very powerful to predict 328 individuals' attitudes toward automation. Trust in automation might be, at least partly, 329 included in individuals' assessments when completing these questionnaires. That being 330 said, trust specific questionnaires are also valuable because trust remains a major 331 influence of operator's reliance in complex and uncertain situations, such as driving, 332 especially with very complex automation, like HAD systems (Lee \& See, 2004).

333 Finally, participants' perceived reputation of HAD systems was a solid predictor of TiAD, 334 confirming results from Kraus et al. (2019). Otherwise, Kraus et al. (2019) found that 335 people whit higher need for cognition relied more on given information about brand or 336 reliability. Here, the openness factor from the Big Five, which is often associated with the 337 need for cognition, did not seem to be a good predictor of TiAD all things equal otherwise. 338 As the present contribution did not manipulate any information prior to the survey, this 339 result is not surprising, but it raises questions about the influence of such personality traits 340 on people's initial learned level of TiAD. 


\section{$341 \quad$ Limitations and perspectives}

342 Culture is one of the several factors linked with dispositional trust. Nevertheless, the 343 survey used in the present study was in French only. Future studies could investigate the 344 topic in other language-speaking areas, to compare results and better understand the 345 effect of culture on TiAD. Next, because HAD systems are currently not fully implemented, 346 drivers' level of TiAD seems strongly influenced by their mental model of such automation.

347 In this context, manufacturers' demonstrations, advertising, reports of accidents and other 348 external stimuli are likely to have a major influence on this mental representation, and 349 therefore on people's level of TiAD. It seems urgent to assess finely the impact of external 350 data on drivers' level of trust and include this supplemental input in predictive models to 351 extend their accuracy. Then, the presented model should be confronted with other data.

352 For example, it may be valuable to test the model with level of trust computed with the 353 Jian et al. (2000)'s scale, in order to confront the model with a general TiA measure.

354 Finally, the present contribution did not assess the Hoff and Bashir (2015)'s situational 355 trust layer. This seemed irrelevant to study drivers' initial level of trust at this point of HAD 356 development, but this trust layer will have to be assessed in the future.

\section{5. CONCLUSION}

358 On top of the assessment of a TiAD specific scale to measure the initial level of trust in 359 Automated Driving, the current contribution offered a modelling of the dispositional and 360 initial learned levels of trust proposed by Hoff and Bashir (2015) within the automotive 361 domain. A general synthesis of the model's findings is available in Figure 6 . The model 362 allows to propose a partial operationalization of the Hoff and Bashir (2015)'s model, 363 adapted to the HAD context. The estimates predicted by the model are indicated for each 
364 factor, allowing to better understand what TiAD is made of. It appears female and people 365 who drive frequently will tend to trust less HAD. People with higher education, who have 366 a positive attitude, expect performances will tend to trust HAD more. Elder people, people 367 who perceive HAD with a positive reputation, and people who are prone to delegate tasks 368 will also trust HAD more. Finally, conscientious people will likely trust HAD less.

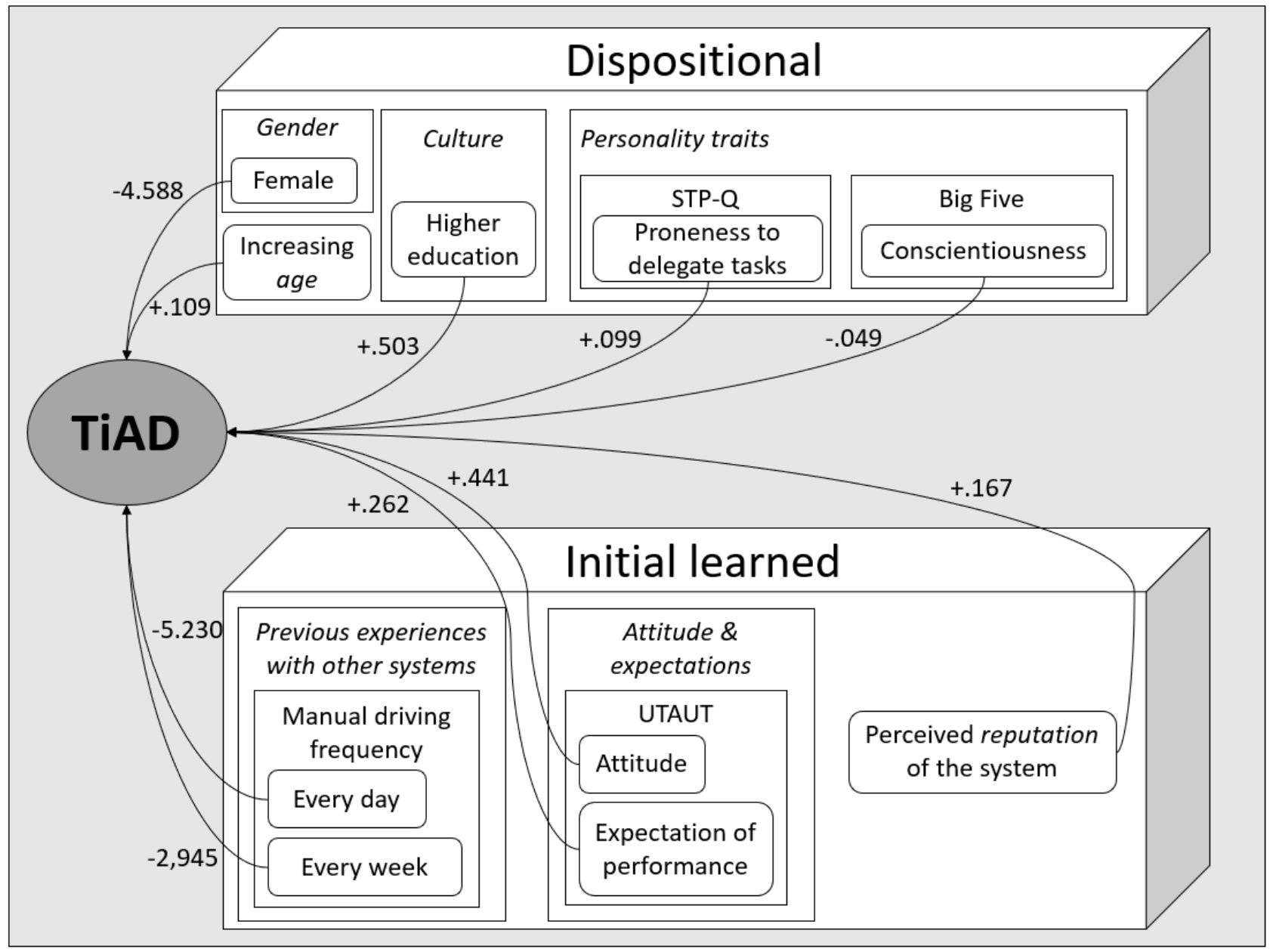

Figure 6. Synthesis of the findings from the linear model. Factors from Hoff and Bashir (2015) are indicated in italic. Estimates calculated by the linear model are indicated near each arrow. 


\section{ACKNOWLEDGMENTS}

371 Funding: This study was supported by VEDECOM Institute, "Institute for Energy

372 Transition" and part of the French governmental plan "Investment for the Future" (ANR-

373 10-IEED-0009).

374 The authors declare that they have no known competing financial interests or personal

375 relationships that could have appeared to influence the work reported in this paper. 


\section{REFERENCES}

377 Agarwal, R., \& Prasad, J. (1998). A conceptual and operational definition of personal 378 innovativeness in the domain of information technology. Information Systems Research, 379 9(2), 204-215. https://doi.org/10.1287/isre.9.2.204

Ayoub, J., Yang, X. J., \& Zhou, F. (2021). Modeling dispositional and initial learned trust in automated vehicles with predictability and explainability. Transportation Research Part F: Traffic Psychology and Behaviour, 77, 102-116. https://doi.org/10.1016/j.trf.2020.12.015

383

384

385

386

387

388

389

390

391

392

393

394

395

396

Benjamini, Y., \& Hochberg, Y. (1995). Controlling the False Discovery Rate: A Practical and Powerful Approach to Multiple Testing. Journal of the Royal Statistical Society: Series B (Methodological), 57(1), 289-300. https://doi.org/10.1111/j.2517-6161.1995.tb02031.x

Brown, T. A. (2015). Confirmatory factor analysis for applied research, 2nd ed (pp. xvii, 462). The Guilford Press.

Carlson, J., \& O'Cass, A. (2012). An empirical assessment of consumers' evaluations of web site service quality: Conceptualizing and testing a formative model. Journal of Services Marketing, 26(6), 419-434. https://doi.org/10.1108/08876041211257909

Costello, A. B., \& Osborne, J. (2005). Best practices in exploratory factor analysis: Four recommendations for getting the most from your analysis. Practical Assessment, Research, and Evaluation, Vol. 10., Article 7. https://doi.org/10.7275/JYJ1-4868

Desrosières, A., \& Thévenot, L. (2002). Les catégories socioprofessionnelles: Vol. 5e éd. La Découverte; Cairn.info. https://www.cairn.info/les-categories-socioprofessionnelles-9782707138569.htm 
Dzindolet, M. T., Peterson, S. A., Pomranky, R. A., Pierce, L. G., \& Beck, H. P. (2003). The role of trust in automation reliance. International Journal of Human-Computer Studies, 58(6), 697-718. https://doi.org/10.1016/S1071-5819(03)00038-7

400

Egea, J. M. O., \& González, M. V. R. (2011). Explaining physicians' acceptance of EHCR systems: An extension of TAM with trust and risk factors. Computers in Human Behavior, 27(1), 319-332. https://doi.org/10.1016/j.chb.2010.08.010

Ekman, F., Johansson, M., \& Sochor, J. (2018). Creating appropriate trust in automated vehicle systems: A framework for HMI design. IEEE Transactions on Human-Machine Systems, 48(1), 95-101. https://doi.org/10.1109/THMS.2017.2776209

Hartwich, F., Witzlack, C., Beggiato, M., \& Krems, J. F. (2018). The first impression counts - A combined driving simulator and test track study on the development of trust and acceptance of highly automated driving. Transportation Research Part F: Traffic Psychology and Behaviour. https://doi.org/10.1016/j.trf.2018.05.012

Hergeth, S., Lorenz, L., \& Krems, J. F. (2017). Prior familiarization with takeover requests affects drivers' takeover performance and automation trust. Human Factors, 59(3), 457-470. https://doi.org/10.1177/0018720816678714

Hergeth, S., Lorenz, L., \& Vilimek, R. (2016). Keep your scanners peeled: Gaze behavior as a measure of automation trust during highly automated driving. Human Factors, 3(58), 509519. https://doi.org/10.1177/0018720815625744

Hoff, K. A., \& Bashir, M. (2015). Trust in automation: Integrating empirical evidence on factors that influence trust.

Human

Factors,

$57(3)$,

407-434. https://doi.org/10.1177/0018720814547570 
Kaur, K., \& Rampersad, G. (2018). Trust in driverless cars: Investigating key factors influencing the adoption of driverless cars. Journal of Engineering and Technology Management, 48, 87-96. https://doi.org/10.1016/j.jengtecman.2018.04.006

Körber, M., Gold, C., Lechner, D., \& Bengler, K. (2016). The influence of age on the take-over of vehicle control in highly automated driving. Transportation Research Part F: Traffic Psychology and Behaviour, 39, 19-32. https://doi.org/10.1016/j.trf.2016.03.002

Körber, M., Prasch, L., \& Bengler, K. (2018). Why do I have to drive now? Post hoc explanations of takeover requests. Human Factors, 3(60), 305-323.

Kraus, J., Forster, Y., Hergeth, S., \& Baumann, M. (2019). Two routes to trust calibration: Effects of reliability and brand information on Trust in Automation. International Journal of Mobile Human Computer Interaction, 11(3), 17.

Kraus, J., Scholz, D., Messner, E.-M., Messner, M., \& Baumann, M. (2020). Scared to trust? Predicting trust in highly automated driving by depressiveness, negative self-evaluations and state anxiety. Frontiers in Psychology, 10. https://doi.org/10.3389/fpsyg.2019.02917

Kraus, J., Scholz, D., Stiegemeier, D., \& Baumann, M. (2020). The more you know: Trust dynamics and calibration in highly automated driving and the effects of take-overs, system malfunction, and system transparency. Human Factors: The Journal of the Human Factors and Ergonomics Society, 62(5), 718-736. https://doi.org/10.1177/0018720819853686

Lee, J. D., \& Moray, N. (1994). Trust, self-confidence, and operators' adaptation to automation. International Journal of Human-Computer Studies, 40(1), 153-184. https://doi.org/10.1006/ijhc.1994.1007

Lee, J. D., \& See, K. (2004). Trust in automation: Designing for appropriate reliance. Human Factors, 46(1), 50-80. https://doi.org/10.1518/hfes.46.1.50_30392 
442 Manchon, J. B., Bueno, M., \& Navarro, J. (2020). From manual to automated driving: How does 443 trust evolve? Theoretical Issues in Ergonomics Science, 1-27. $444 \quad$ https://doi.org/10.1080/1463922X.2020.1830450

445 Manchon, J. B., Bueno, M., \& Navarro, J. (2021). Calibration of Trust in Automated Driving: A 446 matter of initial level of trust and automated driving style? Human Factors, (accepted).

447 Manchon, J. B., Bueno, M., \& Navarro, J. (2022). How the initial level of trust in automated driving impact drivers' behavior and early trust construction. Transportation Research Part F: Traffic Psychology and Behaviour, (accepted).

Marsh, S., \& Dibben, M. R. (2003). The role of trust in information science and technology. Annual 451 Review of Information Science and Technology, 37(1), 465-498.

Molnar, L. J., Ryan, L. H., Pradhan, A. K., Eby, D. W., St. Louis, R. M., \& Zakrajsek, J. S. (2018). Understanding trust and acceptance of automated vehicles: An exploratory simulator study of transfer of control between automated and manual driving. Transportation https://doi.org/10.1002/aris.1440370111

Navarro, J. (2019). A state of science on highly automated driving. Theoretical Issues in Ergonomics Science, 20(3), 366-396. https://doi.org/10.1080/1463922X.2018.1439544

Payre, W. (2015). Conduite complètement automatisée: Acceptabilité, confiance et apprentissage de la reprise de contrôle manuel [Unpublished psychology thesis, Université Paris 8]. https://www.theses.fr/2015PA080115 practice on manual control recovery. Human Factors, 58(2), 229-241. https://doi.org/10.1177/0018720815612319 
466

467

468

469

470

471

472

473

474

475

476

477

478

479

480

481

482

483

484

485

486

487

Plaisant, O., Courtois, R., Réveillère, C., Mendelsohn, G., \& John, O. P. (2010). Validation par analyse factorielle du Big Five Inventory français (BFI-Fr). Analyse convergente avec le NEO-PI-R. 168(2), 97-106.

R Core Team. (2017). R: A Language and Environment for Statistical Computing. R Foundation for Statistical Computing. https://www.R-project.org/

Revelle, W. (2020). psych: Procedures for personality and psychological research (R package version 2.0.8) [Computer software]. North-western University, Evanston.

Rice, S. (2009). Examining single-and multiple-process theories of trust in automation. The Journal of General Psychology, 136(3), 303-322.

Rosseel, Y. (2012). lavaan: An R Package for Structural Equation Modeling. Journal of Statistical Software, 48(2). https://doi.org/10.18637/jss.v048.i02

SAE International. (2016). Taxonomy and Definitions for Terms Related to Driving Automation Systems for On-Road Motor Vehicles. https://doi.org/10.4271/J3016_201609

Schneider, T., Jessup, S., Stokes, C., Rivers, S., Lohani, M., \& McCoy, M. (2017). The influence of trust propensity on behavioral trust. Poster session presented at the meeting of Association for Psychological Society, Boston.

Shin, K. J., Tada, N., \& Managi, S. (2019). Consumer demand for fully automated driving technology. Economic Analysis and Policy, 61, 16-28. https://doi.org/10.1016/j.eap.2018.10.002

Venkatesh, V., Morris, M. G., Davis, G. B., \& Davis, F. D. (2003). User Acceptance of Information Technology: Toward a Unified View. MIS Quarterly, 27(3), 425. https://doi.org/10.2307/30036540 
488 Walker, F., Wang, J., Martens, M. H., \& Verwey, W. B. (2019). Gaze behaviour and electrodermal activity: Objective measures of drivers' trust in automated vehicles. Transportation Research Part F: Traffic Psychology and Behaviour, 64, 401-412. https://doi.org/10.1016/j.trf.2019.05.021

492 Zhang, T., Tao, D., Qu, X., Zhang, X., Lin, R., \& Zhang, W. (2019). The roles of initial trust and 493 perceived risk in public's acceptance of automated vehicles. Transportation Research Part 494 C: Emerging Technologies, 98, 207-220. https://doi.org/10.1016/j.trc.2018.11.018 